Provided by the author(s) and University of Galway in accordance with publisher policies. Please cite the published version when available.

\begin{tabular}{|c|l|}
\hline Title & $\begin{array}{l}\text { Wireless transfer of images from a digital camera to the } \\
\text { internet via a standard GSM mobile phone }\end{array}$ \\
\hline Author(s) & Corcoran, Peter M.; Bigioi, Petronel \\
\hline $\begin{array}{c}\text { Publication } \\
\text { Date }\end{array}$ & 2001 \\
\hline $\begin{array}{c}\text { Publication } \\
\text { Information }\end{array}$ & $\begin{array}{l}\text { P. Corcoran, P. Bigioi, E. Steinberg (2001) " Wireless transfer } \\
\text { of images from a digital camera to the internet via a standard } \\
\text { GSMobile phone", IEEE Transactions on Consumer } \\
\text { Electronics, Vol. 47, No. 3, pp. 542-547. }\end{array}$ \\
\hline Publisher & IEEE \\
\hline Item record & http://hdl.handle.net/10379/283 \\
\hline
\end{tabular}

Some rights reserved. For more information, please see the item record link above. 


\title{
WIRELESS TRANSFER OF IMAGES FROM A DIGITAL CAMERA TO THE INTERNET VIA A STANDARD GSM MOBILE PHONE
}

\author{
Peter M. Corcoran ${ }^{1}$, Petronel Bigioi ${ }^{1}$ and Eran Steinberg ${ }^{2}$ \\ ${ }^{1}$ FotoNation Ireland Ltd., No. 3 Liosban Business Park, Tuam Road, Galway, Ireland \\ ${ }^{2}$ Zing Networks Inc., Bryant St., San Francisco, California
}

\begin{abstract}
In this paper we describe an infrastructure based on an existing, commercially available, GSM mobile phone for achieving connectivity between digital cameras and an Internet Website. Pictures are loaded from a standard digital camera, or compact flash card, by an embedded appliance, which establishes the Internet connection and controls the transfer of pictures from the camera to a remote website. Detailed descriptions are given of this picture transfer appliance and of a typical website infrastructure to store and manage the pictures and provide end-user services.
\end{abstract}

\section{Introduction}

Today's digital cameras are increasingly sophisticated and are rapidly superseding conventional photography. However a digital camera does not offer consumers the ease of use and service available from conventional photography today. In particular it is difficult and expensive for consumers to obtain prints of their digital images. In addition, they must rely on their home PC for long-term storage of such images. In short the digital photography solutions available today are inadequate for the needs of many consumers.

In this paper we describe how providing wireless Internet connectivity can extend the range of services available to consumers using digital cameras. We examine how today's consumer uses digital images and describe how wireless connectivity solutions can be provided in today's marketplace by extending the functionality of a commercially available GSM mobile phone using an inexpensive embedded appliance.

Mobile data networks will soon begin the transition to 3rd generation technology - commonly known as G3. However, in the current marketplace it is likely that the capital investment for $\mathrm{G} 3$ infrastructure will be difficult to raise for another few years. This is likely to delay the development of G3 by 1-2 years. Thus it becomes important for network operators to investigate new data services over existing networks.

In this paper we describe our experiences in implementing just such a data service - the transfer of digital pictures from a standard digital camera or compact flash memory card to a remote Internet site using existing GSM networks and mobile phones. Once the pictures are transferred onto the website the end-user can access a broad range of added-value services for their pictures [1].

\section{Elements of a Mobile Photography Service}

We now describe the component parts of a fully integrated mobile digital photography service. Note that all these components exists today and that the service proposed in this paper can be implemented with today's technology and existing mobile infrastructure.

\subsection{Digital Picture Sources}

The first component is, naturally enough, a source of digital pictures. In most instances this will be a digital camera, although many users of the service may prefer the simpler option to use compact flash (CF) memory cards to store and manage the pictures.

Digital cameras are now quite ubiquitous. The more recent models all feature USB connectivity as the primary means of moving the pictures and other content such as audio and video clips from the camera. Older models use RS-232 although this can be quite slow if the digital images are larger than a 50k JPEG - typically $640 \times 480$ VGA size. However we note that it may not be desirable to send larger pictures over a wireless GSM network as even recent enhancements to existing networks can rarely achieve $38 \mathrm{kB}$ throughput. Further some mobile phones provide data connectivity at RS-232 compatible data rates but we have not yet seen a mobile phone to offer USB connectivity or data rates. Thus for the pilot study described in this paper we have confined our connectivity support of current digital cameras to access over RS-232.

As many users now make use of CF cards to store and transport their "rolls" of digital pictures it was also felt important to provide support for the direct upload of digital images from such portable media. Most digital cameras now rely entirely on a CF card to store the final images and models exist with up to $256 \mathrm{MB}$ of storage.

\subsection{GSM Phone \& Data Infrastructure}

Until recently it was possible; although slow and expensive, to obtain data services using a GSM mobile 
phone. With the advent of G3 services many users were happy to postpone the transition to mobile services until G3 roll-out occurred. For similar reasons GSM service operators did not invest time or effort in trying to upgrade or improve existing data services. It is widely anticipated that as next generation G3 services come on stream Internet services will initially be offered at $115 \mathrm{k}$ baud rates. This is expected to rise to $384 \mathrm{k}$ baud within 12 months of initial deployment. Ultimately, it is expected that some next generation GSM services will support wireless 2-Megabit connectivity to the Internet. These new wireless services will undoubtedly be one of the key driving forces of Internet Photography over the next 2-3 years.

As it is now becoming widely accepted that G3 roll-out has been postponed due to the prohibitive costs and uncertain market for services there is a renewed demand for faster data services based on existing network infrastructure.

One alternative is GPRS with planned availability for many existing networks by early 2002 . In the meantime there are other approaches - one handset manufacturer has released mobile phones with 3 -speed modems. These are capable of providing 3 GSM channels with a resulting throughput of $3 \times 14.4 \mathrm{kB}$ if the service operator does not implement error correction or $3 \times 9.6 \mathrm{kB}$ if error correction is implemented. Thus theoretical data rates of $43.2 \mathrm{kB}$ or $28.8 \mathrm{kB}$ are possible using existing mobile networks available, and in widespread usage, on a day-today basis.

For the pilot study described in this paper we used a 3speed modem to provide mobile internet access. We expect that the same technology could be readily adapted to work with GPRS and G3 data services when they become widely available.

\subsection{Embedded Picture Transfer Appliance}

The core connectivity between the digital camera and mobile phone is provided by an embedded picture transfer appliance (EmPicTA). This device incorporates an RS-232 port to connect directly to a conventional digital camera and a compact flash slot to allow pictures to be read directly from digital media. The connection to the mobile phone is achieved via a proprietary data cable.

The appliance runs an embedded form of DOS. A full TCP/IP stack is implemented in software. In addition a generic interface is provided to a broad range of digital cameras via a modular set of device drivers. An additional, unique, feature of this appliance is that it may be reprogrammed from the Web. As the main functionality of the appliance requires it to connect to the Internet it searches, by default, for a home web page.
Upon accessing this page the appliance determines if its firmware is up to date. If not, it will load a new firmware mask into its flash memory. This feature allows, for example, additional camera drivers to be easily added to the appliance in a manner transparent to the user.

Internet connectivity is achieved by establishing a PPP link via the mobile phone network. The unit is designed to connect to any standard ISP.

\subsection{Website Services for Digital Photography}

To support the client appliance it is important that a flexible server-side infrastructure exists to provide the end user with a range of exciting and interesting new services, focused around the network-enabled digital camera.

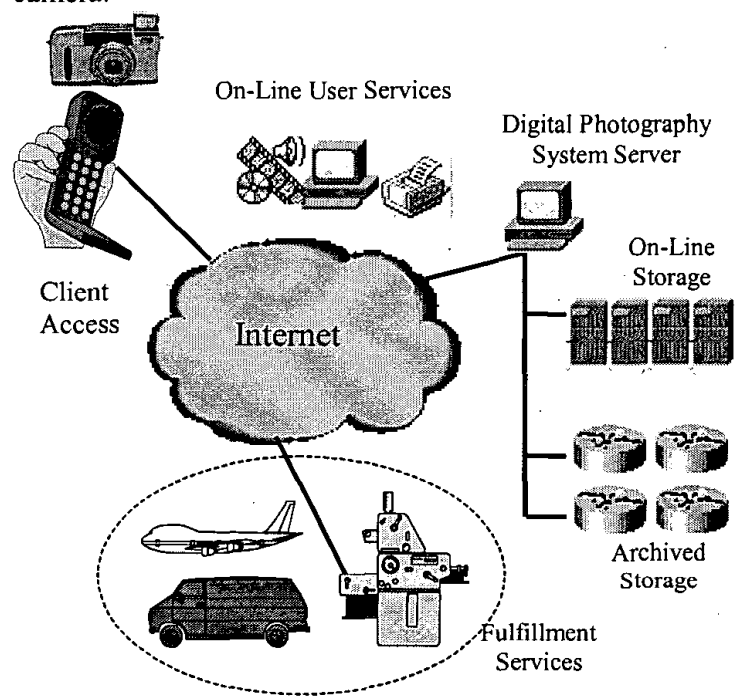

Fig 1: Diagram illustrating the various web services required by most users of an Internet Photo Community. .

The key services that will initially be required in order to promote the success of digital photography on a massmarket scale are:

(i) picture fulfillment services

(ii) on-line picture storage

(iii) archival picture storage

(iv) added-value on-line services

In an experimental laboratory set up these services can be adequately provided by a conventional PC-based web server with a large hard disk to provide on-line storage and a tape drive to provide archival storage facilities. Added value services can be programmed using one of the high-level scripting languages that are popular for web development.

Note that a key aspect of this client-server architecture for digital photography is that practically all of the client configuration information can be maintained on the 
server-side. This, in turn, reduces maintenance issues and facilitates upgrading of services and the infrastructure as a whole.

In Fig 2 we show how the user can access the pictures on a remote website. Some of this functionality could also be made available from the mobile phone through a WAP or iMode service.

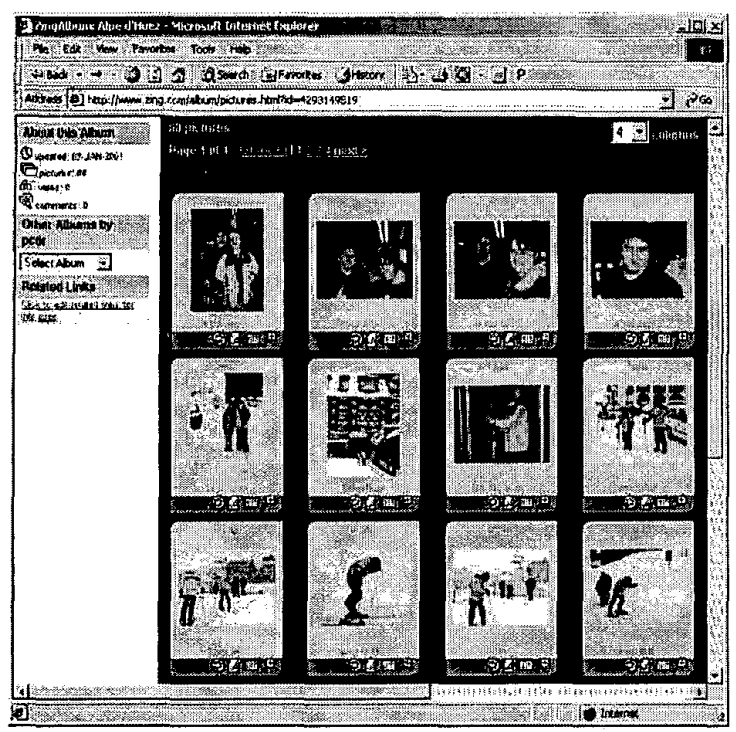

Fig 2(a) Picture access using a standard Web Browser.

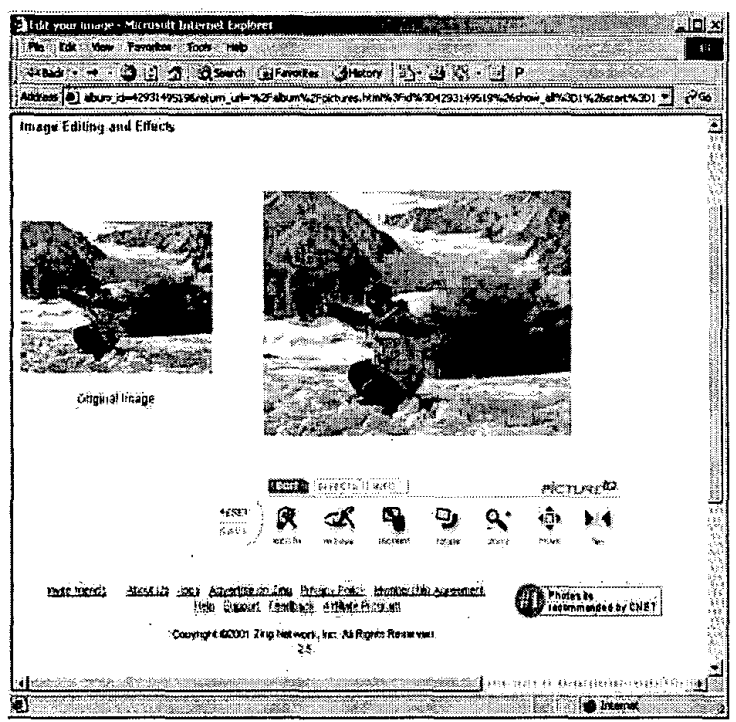

Fig 2(b) Picture editing and post-processing tools are available via the Web interface.

\section{Implementing the Service}

In this section we give some details of our experimental implementation of an internet-enabled digital photography system. Some descriptions of the system services and overall system infrastructure have already been given, so here we will focuss on practical implementation issues.

\subsection{An Initial Approach}

As a first step towards investigating the practicality of building a service solution for GSM we undertook a project to demonstrate Direct-to-Web services using the existing GSM infrastructure. For this technology demonstration we decided to use a standard and very well known GSM PDA. This device combines a mobile phone with a full alphanumeric keyboard and a reasonably sized grayscale LCD. It has an RS-232 port and can establish an Internet connection over the GSM network to allow the user to receive e-mail or to browse the Web.

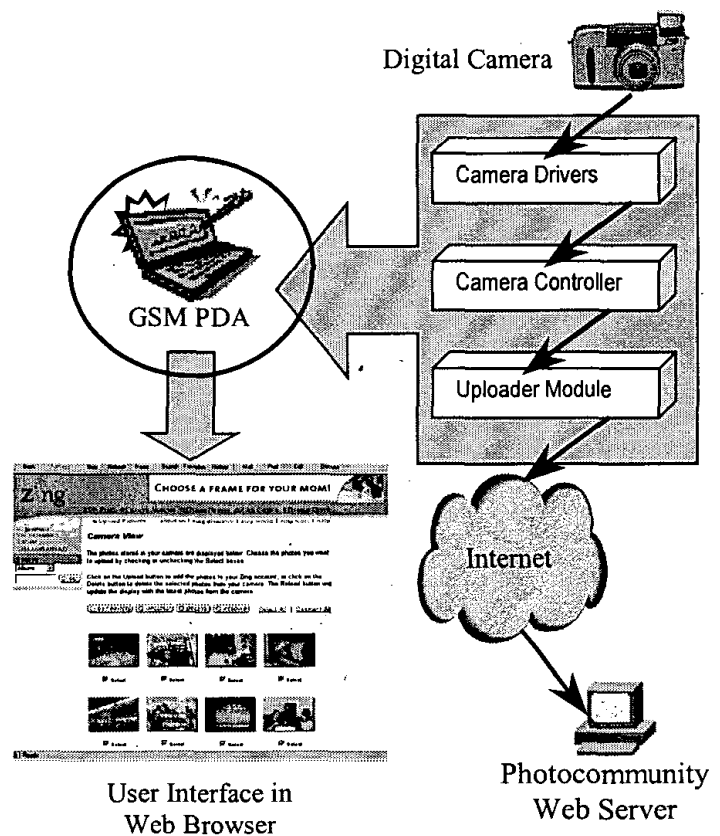

Fig 3: The component elements of a Home-PC based Directto-Web Interface for Digital Cameras.

This GSM PDA runs a windowing OS known as GEOS. This is an older OS, originating on simple x86 systems, but it provides adequate multi-tasking functionality to allow us to implement a Direct-to-Web solution for a standard digital camera.

This service solution is, naturally enough, somewhat cruder than its big-brother equivalent which runs on a 
standard Pentium-based PC. For example, only filenames are displayed when the user has to select the pictures for uploading to the Web. It is just not practical to provide a thumbnail view to the end user. Further the data transfer rate is limited to 9600 baud over the GSM connection which limits the practicality utility of this demonstration. However the key point is that it is possible to port the multi-camera Web solution which runs on today's Pentium PC to run on a much less powerful PDA platform.

Fig 3 illustrates the PDA solution. On the RHS of the diagram we see that software drivers implemented on the PDA connect with the digital camera. The driver software inter-operates with a camera controller daemon which, in turn, inter-operates with a third software module which controls the upload of pictures/data to a back-end server. The complex operations involved in obtaining pictures from the camera and uploading them to the back-end server are transparent to the end-user who sees a single, unified interface presented in a web browser - the LHS of the diagram.

More recently we have completed an initial port of our picture upload technology onto the EPOC operating system. This will form the core OS to be adopted by next-generation mobile appliances. Already many nextgeneration mobile PDA's and "smart" phones use EPOC as their OS. It provides a more advanced windowing environment than GEOS and is capable of handling mouse or stylus input from a touch-screen. Thus it is feasibly to retain many aspects of the user interface and the features of the desktop PC application, but operating on a much smaller mobile appliance.

\subsection{The EmPic'TA Prototype}

Following our implementation of a PDA-centric port it was decided to investigate the feasibility of providing a dedicated appliance, the EmPicTA, to work with a conventional mobile phone. Clearly the user interface would be much simpler and the concept was initially to use the phone solely as a "picture uploading" conduit; additional services would be provided via a Web browser or WAP services independently of the picture upload process.

\subsubsection{EmPicTA Hardware}

Here we give an overview description of our actual IIA client hardware. As was mentioned earlier, this hardware is limited to RS-232 camera connectivity and to analogmodem Internet connectivity. The Internet connectivity is achieved either over a PPP link or via a direct, non-IP link to a middleware server, which provides an internet relay service.
The layout of the hardware is illustrated in fig 4 below. The system has both RAM and flash memory. The flash has a boot area, which can be dynamically reprogrammed, and a disk area, which appears like a DOS compatible disk to the operating system.

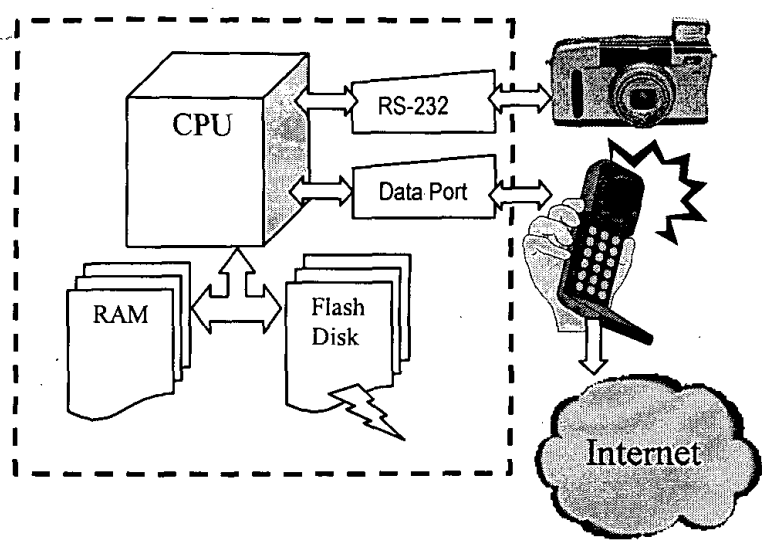

Fig 4: Block diagram of the Embedded Picture Transfer Appliance (EmPicTA). An Internet connection is established over the data port connection of the mobile phone.

\subsubsection{EmPicTA Client-End Software}

The EmPicTA client runs an embedded OS that is compatible with DOS. Depending on the client-server architecture the IIA client may run either a TCP/IP stack and PPP or it may run a more lightweight non-IP data transfer program.

From our experiences there is significant overhead in implementing a full TCP/IP stack on the client. Furthermore as TCP/IP does not, in its present form, guarantee any quality of service (QOS) it is conceivable that picture download over a slow link-will be both timeconsuming and costly for the consumer. If a lightweight non-IP transfer program is used then practically all the limited modem bandwidth can be devoted to transferring picture data. This offers a $30-40 \%$ performance improvement over the best case for TCP/IP.

The client also runs an embedded camera protocol module with establishes a connection with the camera and downloads the "digital film" from the camera. The EmPicTA can be easily reprogrammed with a wide range of camera protocols, or these may be loaded dynamically from the internet-photography server.

\subsubsection{The Internet Infrastructure}

Mention was already made of the difference between the EmPicTA client-server model and more conventional client-server models. The EmPicTA client is, in a sense, 
a micro-server and this introduces some complications that are unique to the EmPicTA client-server model. Furthermore the EmPicTA client will suffer from bandwidth limitations when used with a GSM phone. This limits the data throughput to the main system server.

To best understand these issues it is useful to study the protocol stack shown in fig 5 below. The key point is that, for a modem-based version of the EmPicTA client hardware there are PPP protocol layers in addition to the standard TCP/IP protocol layer. The PPP guarantees robust communications, but when large block of digital data, such as digital pictures are transmitted through such a protocol stack there is a significant overhead. In our best-case studies between $30 \%$ and $40 \%$ of the transmission bandwidth is lost to such protocol overheads. Note that there is less overhead in an Ethernet based stack.

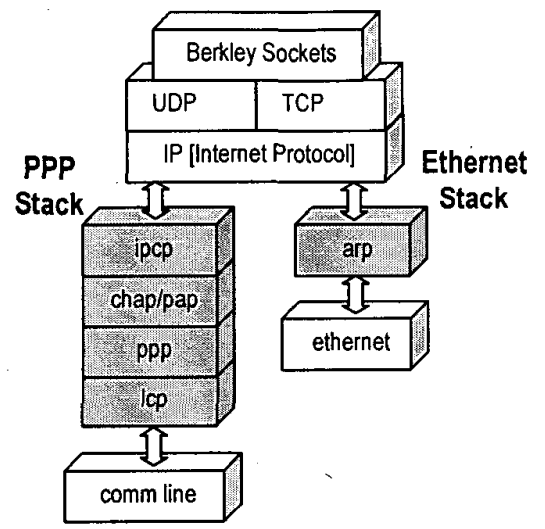

Fig 5: Protocol Stacks for EmPicTA Client.

This form of EmPicTA client has a complete TCP/IP solution integrated into the basic appliance hardware. Thus the EmPicTA client connects a digital camera directly to the Internet over the modem/PPP link and digital pictures are sent directly to the main system server over this TCP/IP link. We call this a two-tier client server solution and the main system infrastructure is illustrated in fig 6(a) below.

There are a number of key drawbacks to this two-tier architecture. Apart from the protocol overhead we must also consider that if the EmPicTA client does not succeed in making a direct, fast connection to the remote system server then there will be additional delays over the Internet connection. This will increase the online time required by the client and if a link proves unreliable then pictures can easily be lost in transit from EmPicTA client to the main server.

A more complex three-tier system architecture [2] overcomes these difficulties and provides a more reliable infrastructure. It also requires less complicated client software on the appliance and allows faster data transfer from the EmPicTA appliance to the Internet. This alternative systems architecture is illustrated in $f i g \mathbf{6}(\boldsymbol{b})$ below.

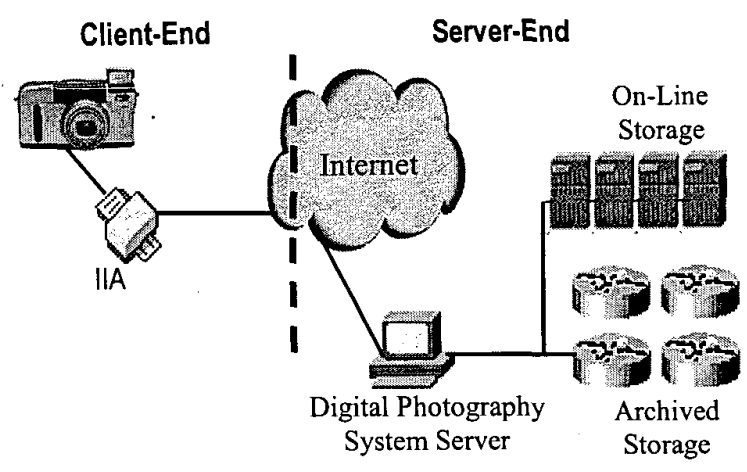

Fig 6(a): The Two-Tier Infrastructure.

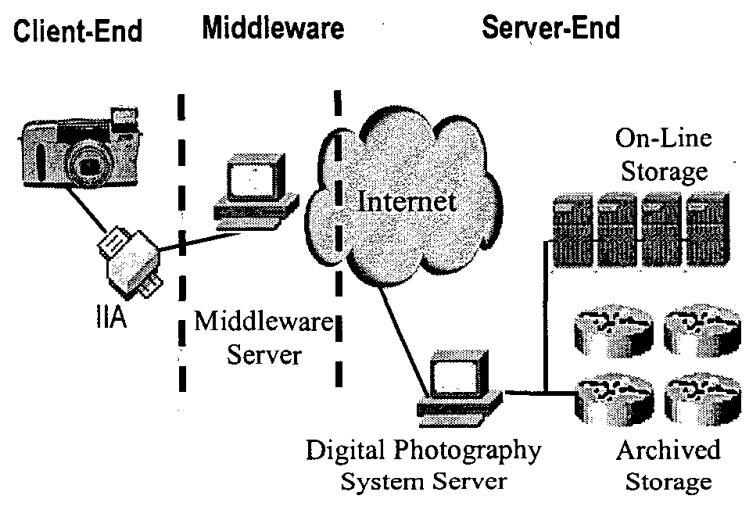

Fig 6(b): The Three-Tier Infrastructure.

This 3-tier architecture has the additional benefit that the middle-tier, or proxy server can forward the images to different photocommunity websites allowing the end-user greater flexibility in their choice of services. It also overcomes the integration issues which often arise in dealing with server-side modifications to large, missioncritical, websites.

\section{Conclusions}

The main purpose of this paper has been to demonstrate that a workable mobile photography service can be offered to consumers with today's technology infrastructure. Recent improvements in data services over GSM have made it possible to offer reasonable upload rates for digital pictures. Services such as WAP and iMode now offer the possibility to access and manipulate uploaded pictures directly from a mobile phone. 
However there are still many barriers to the broad adoption of such services. Our experimental service requires a dedicated upload appliance - the EmPicTA - to access a digital camera and establish a connection with the Internet. Until such functionality is integrated directly into a mobile phone unit it will be necessary for an-enduser to purchase such a dedicated peripheral. Further, as the current length. of time to upload a standard $16 \mathrm{MB}$ compact flash card of pictures is of the order of several hours such a service will be very expensive to use unless mobile operators market it in an imaginative manner. One approach we have proposed is to make the picture upload service available at night time when mobile networks carry very little traffic and a user could be offered a bulk billing rate. An additional problem is that mobile phone batteries will generally only sustain a 2 hour talk-time when fully charged. To overcome both problems we have proposed developing a combined battery-charger and picture/data upload unit.

Fig 7 shows an artists impression of a production version of such a picture-upload and battery-charging cradle for a mobile phone. We believe that such a connectivity appliance, as described in this paper provide a practical, market-focussed, bridge between the Internet and digital cameras and mobile phones available in today's market. This illustrates that wireless digital photography is a service, which could be made available to consumers today and that it is no longer a dream of tomorrow.

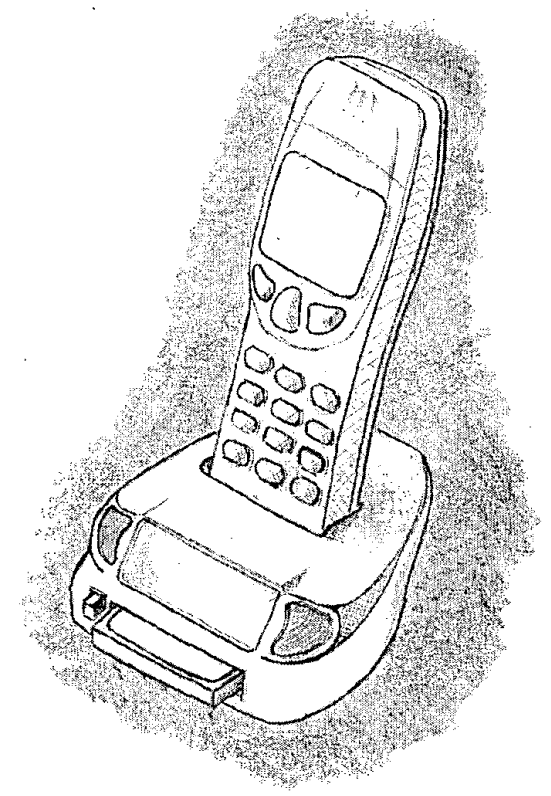

Fig 7: Artist's concept sketch of a combined battery-charger and photo-upload appliance

\section{References}

[1] Corcoran P.M., and Steinberg, E., "Internet Connectivity Solutions for Digital Photography", IEEE Transactions on Consumer Electronics, Vol. 46 No. 3 p.494-498, Aug. 2000.

[2] Corcoran, P.M., Bigioi, P., Steinberg, E. and Prilutsky, Y., "Internet Enabled Digital Photography", IEEE Transactions on Consumer Electronics, Vol. 45, No. 3, 1999, pp. 577-584, Nov 1999

\section{Biographies}

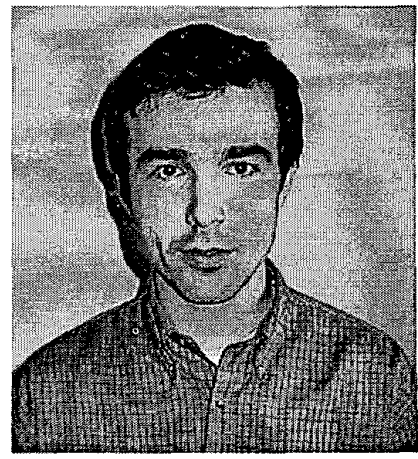

Petronel Bigioi received his B.S. degree in Electronic Engineering from "Transilvania" University Brasov, Romania, in 1997. At the same university he received in 1998 M.S. degree in Electronic Design Automation. He received a M.S. degree in electronic engineering at National University of Ireland, Galway in 2000. Currently he is working as R\&D Engineer with FotoNation Ireland, a Zing Networks subsidiary. His_ research interests include VLSI design, communication network protocols and embedded systems.

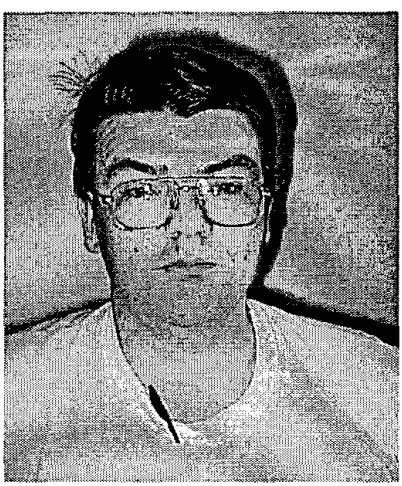

Peter Corcoran received the BAI (Electronic Engineering) and $\mathrm{BA}$ (Math's) degrees from Trinity College Dublin in 1984. He continued his studies at TCD and was awarded a Ph.D. in Electronic Engineering for research work in the field of Dielectric Liquids. In 1986 he was appointed to a lectureship in Electronic Engineering at UCG. His research interests include microprocessor applications, environmental monitoring technologies. He is a member of I.E.E.E. 